\title{
Quantification of three-dimensional computed tomography angiography for evaluating coronary luminal stenosis using digital subtraction angiography as the standard of reference
}

Wei Guo ${ }^{1 \dagger}$, Xin Liu' 2,3†, Zhifan Gao 2,3,6†, Sandeep Pirbhulal2,3,6, Wenhua Huang ${ }^{4}$, Wan-Hua Lin ${ }^{2,3 *}$, Heye Zhang ${ }^{2,3}$, Ning $\operatorname{Tan}^{1}$ and Yuan-Ting Zhang ${ }^{2,3,5}$

\section{${ }^{*}$ Correspondence:}

wh.lin@siat.ac.cn

†Wei Guo, Xin Liu and Zhifan

Gao contributed equally to this work.

${ }^{2}$ Institute of Biomedical and Health Engineering,

Shenzhen Institutes

of Advanced Technology,

Shenzhen 518055, China

Full list of author information

is available at the end of the article

\begin{abstract}
Objective: We sought to evaluate the accuracy of quantitative three-dimensional (3D) CT angiography (CTA) for the assessment of coronary luminal stenosis using digital subtraction angiography (DSA) as the standard of reference.

Method: Twenty-three patients with 54 lesions were referred for CTA followed by DSA. The CTA scans were performed with 256-slice spiral CT. 3D CTA were reconstructed from two-dimensional CTA imaging sequences in order to extract the following quantitative indices: minimal lumen diameter, percent diameter stenosis (\%DS), minimal lumen area, and percent area stenosis (\%AS). Correlation and limits of agreement were calculated using Pearson correlation and Bland-Altman analysis, respectively. The diagnostic performance and the diagnostic concordance of 3D CTAderived anatomic parameters (\%DS, \%AS) for the detection of severe coronary arterial stenosis (as assessed by DSA) were presented as sensitivity, specificity, diagnostic accuracy, and Kappa statistics. Of which vessels with \%DS >50\% or with \%AS >75\% were identified as severe coronary arterial lesions.
\end{abstract}

Result: The correlations of the anatomic parameters between 3D CTA and DSA were significant $(r=0.51-0.74, \mathrm{P}<0.001)$. Bland-Altman analysis confirmed that the mean differences were small (from -1.11 to $27.39 \%$ ), whereas the limits of agreement were relatively wide (from \pm 28.07 to $\pm 138.64 \%)$. Otherwise, the diagnostic accuracy $(74.1 \%$ with $58.3 \%$ sensitivity and $86.7 \%$ specificity for DS\%; $74.1 \%$ with $45.8 \%$ sensitivity and $96.7 \%$ specificity for \%AS) and the diagnostic concordance ( $k=0.46$ for DS\%; 0.45 for $\% \mathrm{AS}$ ) of 3D CTA-derived anatomic parameters for the detection of severe stenosis were moderate.

Conclusion: 3D advanced imaging reconstruction technique is a helpful tool to promote the use of CTA as an alternative to assess luminal stenosis in clinical practice.

Keywords: Three-dimensional (3D) CT angiography, Digital subtraction angiography, Coronary luminal stenosis 


\section{Background}

Atherosclerotic plaque leads to progressively increasing luminal stenosis, which could result in fatal cardiac events. Coronary angiography is currently the gold standard technique for assessment of coronary lumen stenosis or occlusion [1-4]. However, it is an invasive procedure. A catheter should be used to insert into the coronary arteries for injecting dye, which would induce discomfort for the patient. Furthermore, it has limitations of differentiating plaque components [5]. Therefore, it is of paramount importance to evaluate the lumen stenosis using non-invasive imaging techniques.

During the past decades, CT angiography (CTA) has become a rapidly developing non-invasive imaging technique, which showed promising application in the identification, visualization and characterization of the coronary artery stenosis [6, 7]. Sun et al. $[8,9]$ have discussed the application of 63-slice CT in the diagnosis of coronary artery stenosis intensively. Munnur et al. [10] reviewed how to identify the coronary atherosclerosis, estimate the plaque progression, assess the chest pain in the emergency department, and evaluate the functional significance of stenosis and the prognostic significance by means of CTA. Sun et al. discussed the dose reduction of CTA and the diagnostic and prognostic values on coronary artery disease. In order to evaluate the ability of CTA to identify atherosclerosis, the performance of coronary CTA has been compared to intravenous ultrasound (IVUS), IVUS with radiofrequency backscatter (IVUS/VH), singlephoton emission CT (SPECT) imaging, or fractional flow reserve (FFR) in many studies [11-15]. Furthermore, more quantitative information was derived from CTA for better diagnosis of coronary artery stenosis. For example, Naganuma et al. [12] compared the quantitative measures [minimal lumen area (MLA), plaque burden, and morphology] derived from CTA to the IVUS, and FFR. One promising technique CTA-derived FFR was developed to evaluate the stenosis inside the coronary artery [16]. From one experiment of the sex differences in the visual-functional mismatch using CTA, Park et al. [17] found that female patients might have higher FFR value for any given stenosis compared with male patients. The diagnostic results of CTA could be easily affected, which produces blooming artifacts leading to high false positive rates of coronary stenosis. Sun et al. [18] tried to overcome the problem caused by the heavy calcification in the coronary artery through the measurement of left coronary bifurcation angle. More quantitative measures could be derived from the CTA data, and the clinical value of these measures was examined in 300 patients [19], or was also compared to IVUS [20]. Until now, the prognostic significance of non-invasive coronary CTA for the quantification of luminal stenosis remains controversial. Some previous works reported that anatomic measurements by noninvasive CTA have relatively poor accuracy for the quantification of stenosis severity [21, 22], and for the prediction of hemodynamically significant stenosis [21, 23]. For examples, Meijboom et al. [21] demonstrated that the correlation of the percent diameter stenosis (\%DS) as determined by CTA and quantitative coronary angiography (QCA) was moderate $(\mathrm{R}=0.53$; $\mathrm{p}<0.001)$, and the diagnostic accuracy (49\%) was weak when CTA was used for the detection of hemodynamically significant stenosis based on FFR $<0.75$. Joshi et al. [22] showed that there was no relation between CTA and QCA measurements of minimal luminal diameter (MLD, $\mathrm{r}^{2}<0.01, \mathrm{P}=0.57$ ) or diameter stenosis (DS, $\mathrm{r}^{2}=0.02, \mathrm{P}=0.31$ ). However, other studies demonstrated the great diagnostic value of CTA in the assessment of luminal stenosis that was estimated 
by invasive imaging techniques of IVUS or catheter coronary angiography $[4,24,25]$. For examples, Youssef et al. [4] illustrated that the positive predictive value (PPV) and negative predictive (NPV) value of CTA for the detection of coronary artery stenosis based on gold standard catheter coronary angiography were 94 and 100\%, respectively. Szilard Voros et al. [25] showed that the correlation of the quantitative measurements between CTA and IVUS were significant $(\mathrm{r}=0.41-0.84, \mathrm{P}<0.001)$.

More recently, by the means of advanced imaging reconstruction technique, the evolvement of quantitative CTA measurements derived from three-dimensional (3D) coronary CTA show potential application in accurately diagnosing intermediate-tosevere coronary arterial lesions [4, 24-27], however, 3D CTA-derived luminal measurements have not been compared with digital subtraction angiography (DSA), which currently is the gold standard for luminal stenosis assessment. Therefore, the purpose of this study was to investigate the diagnostic value of 3D quantitative CTA in the assessment of coronary stenosis using DSA as the standard of reference in patients with interpretable coronary arterial stenosis.

\section{Methods}

\section{Study population}

The ethics committee of Guangdong General Hospital approved this retrospective study and written informed consent was obtained from all the patients or relatives before collecting their data. The data acquisition of DSA and CTA were performed in the Department of Cardiology and Department of Medical Imaging, respectively at Guangdong General Hospital. Two experienced cardiologists with 10-year experience would check the condition of each patient who was diagnosed with coronary artery disease (CAD), and suitable patients were directed to perform CTA examination. The patients with CAD confirmed by the CTA were then directed to perform DSA examination. Patients with previous coronary bypass grafts and those with coronary stents were excluded. The study population consisted of 23 patients who completed both the CTA and DSA examination from 30th August 2012 to 20th May 2014. The ages of the patients were from 42 to 81 years $(62.0 \pm 11.9)$.

\section{Measurements}

\section{CT coronary angiography}

The subject was scanned with a 256-slice spiral CT (BriUiance iCT; Philips Healthcare, Cleveland, OH, USA). A bolus of contrast agent $80 \mathrm{~mL}$ (Ultravist 370; Schering, Berlin, Germany) and saline water $30 \mathrm{~mL}$ was injected intravenously at a flow rate of $4.5 \mathrm{~mL} / \mathrm{s}$. The scan started in $5 \mathrm{~s}$ after a threshold of $150 \mathrm{HU}$ was reached in a region of interest positioned in the ascending aorta. We used a contrast agent bolus tracking method. During the scanning, prospective ECG gating was used if the patient's heart rate was $<75$ beats/min, and retrospective ECG gating was used if the patient's heart rate was $>75$ beats $/ \mathrm{min}$. Tube voltage was $120 \mathrm{kV}$, tube current was adjusted by body size [28], gantry rotation $270 \mathrm{~ms}$, and pitch 0.18 . Images were reconstructed at $5 \%$ interval. The reconstruction parameters were set as follows: the section thickness was $0.9 \mathrm{~mm}$, reconstruction interval $0.45 \mathrm{~mm}$, matrix size $512 \times 512$, field of view (FOV) $250 \mathrm{~mm}$. Those two-dimensional (2D) imaging sequences were then transferred to another computer for 3D anatomy reconstruction. 


\section{Digital subtraction angiography}

Invasive DSA was performed based on standard institutional protocols by X-ray angiographic equipment (Allura Xper FD10 System, Philips Healthcare, Netherland). The projection data acquisition was performed with five views of the left coronary, two views of the right coronary, and two orthogonal views of the target lesion. Once the lesion was identified from DSA data, we performed at least two sets of projection data of DSA in two orthogonal views.

\section{Quantification of stenosis severity}

An experienced cardiologist with 10-year experience analyzed the DSA data sets manually. Lumen segments with clearly visible coronary arterial stenosis were marked and measured by hand in the DSA images. MLD and MLA were measured in the view with the greatest degree of the stenosis. Proximal reference diameter (PRefD) and distal reference diameter (DRefD) were then measured and averaged to calculate \%DS. Correspondingly, proximal reference area (PRefA) and distal reference area (DRefA) were measured and averaged to calculate percent area stenosis (\%AS). \%DS, \%AS were calculated as the following formulas:

$$
\begin{aligned}
& \% \mathrm{DS}=1-\mathrm{MLD} /[\mathrm{PRefD}+\mathrm{DRefD} / 2] \times 100 \% \\
& \% \mathrm{AS}=1-\mathrm{MLA} /[\mathrm{PRefA}+\mathrm{DRefA} / 2] \times 100 \%
\end{aligned}
$$

Another interventional radiologist with 7-year experience supervised the analysis of CTA data. The 3D CTA data were reconstructed over Mimics software (Materialise NV, Belgium) based on standard procedures as follows: First of all, coronary arterial mask construction: coronary arteries were identified from the CTA imaging sequences based on a threshold range which were set according to the different gray values of artery and other issue. Second, 3D modeling: a procedure of 3D calculating was performed with the coronary arterial masks. After that, MLD, PRefD, DRefD, MLA, PRefA and DRefA were measured in the corresponding anatomical position as marked in angiographic data sets. $\% \mathrm{DS}$ and \%AS were calculated according to the above-mentioned formulas.

\section{Statistical analysis}

The statistical analysis was performed using SPSS (IBM Company, USA). Continuous variables were expressed as mean $\pm \mathrm{SD}$, while categorical variables were expressed as absolute numbers and percentages. The correlation of anatomic measurements (MLD, \%DS, MLA, \%AS) between CTA and DSA was assessed by Pearson correlation coefficient and linear correlation. A p value of 0.05 or less was considered statistically significant correlation. Mean differences and limits of agreement of anatomic measurements (MLD, \%DS, MLA, \%AS) by CTA and DSA were analyzed using Bland-Altman [29]. Since patients with severe coronary arterial stenosis were suggested to undergo intensive therapy. The diagnostic performance of 3D CTA-derived anatomic parameters (\%DS, \%AS) for the detection of severe coronary arterial stenosis (as assessed by DSA) was presented as sensitivity, specificity, diagnostic accuracy, positive predictive value, and negative predictive value with the corresponding 95\% confidence intervals. The severity of coronary arterial lesions were classified with \%DS and \%AS standard, respectively. Positive was defined as 
vessels with severe coronary arterial stenosis ( $\% \mathrm{DS} \geq 50 \%$ or $\% \mathrm{AS} \geq 75 \%$ ). Negative was defined as vessels without severe coronary arterial stenosis (\%DS $<50 \%$ or $\% \mathrm{AS}<75 \%$ ). True positives (TP) were defined as vessels with severe coronary arterial lesions evaluated both by 3D CTA and DSA. True negatives (TN) were defined as vessels without severe coronary arterial lesions evaluated both by 3D CTA and DSA. False negatives (FN) were defined as vessels without severe coronary arterial lesions evaluated by 3D CTA-derived \%AS or DS\% but with severe coronary arterial lesions evaluated by DSA-derived \%AS or DS\%. False positives (FP) were defined as vessels with severe coronary arterial lesions evaluated by 3D CTA-derived \%AS or DS\% but without severe coronary arterial lesions evaluated by DSA-derived \%AS or DS\%. Sensitivity (Sen, true positive rate) was the calculated as TP/(TP + FN). Specificity (Spe, true negative rate) was calculated asTN/(FP + TN). Positive predictive value was calculate as TP/(TP + FP). Negative predictive value was calculate as TN/(FN + TN). The diagnostic concordance by 3D CTA-, and DSA-derived anatomic parameters for the detection of vessels with and without severe stenosis calculated as kappa statistics.

\section{Results}

General demographic features of the population are listed in Table 1. Two patients had previous myocardial infarction (MI) and no one had coronary artery bypass graft (CABG). A total 54 lesions were interpretable by both 3D CTA and DSA in the 23 patients. Distributions of the coronary lesions in the patients are also listed in Table 1 . Of the 54 interpretable lesions, 26 (48.1\%) lesions distributed in the left anterior descending artery (LAD), 13 (24.1\%) in the right coronary artery (RCA), 8 (14.8\%) in the left circumflex artery (LCX),

Table 1 General demographic parameters and lesions distribution

\begin{tabular}{ll}
\hline Characteristics & Frequency or value \\
\hline Subjects (n) & 23 \\
Age (years) & $62.0 \pm 11.9$ \\
SBP (mmHg) & $131.1 \pm 13.6$ \\
TC (mmol/L) & $4.6 \pm 1.5$ \\
LDL (mmol/L) & $2.7 \pm 1.2$ \\
Men & $13(56.5 \%)$ \\
Chinese people & $23(100 \%)$ \\
Hypertension & $6(26.1 \%)$ \\
Diabetes & $5(21.7 \%)$ \\
Hyperlipidemia & $9(39.1 \%)$ \\
Smoke & $6(26.1 \%)$ \\
Previous Ml & $2(8.7 \%)$ \\
CABG & $0(0.0 \%)$ \\
Lesions distribution & $54(100 \%)$ \\
LAD & $26(48.1 \%)$ \\
RCA & $13(24.1 \%)$ \\
LCX & $8(14.8 \%)$ \\
LM & $5(9.3 \%)$ \\
D1 & $1(1.9 \%)$ \\
OM1 & $1(1.9 \%)$ \\
\hline
\end{tabular}

SBP systolic blood pressure. 
5 (9.3\%) in the left main coronary artery (LM), 1 (1.9\%) in the first diagonal (D1), and 1 (1.9\%) in the first obtuse marginal (OM1). Examples of a same coronary arterial lesion detected by DSA, 2D CTA and 3D CTA are shown in Figure 1. A coronary arterial segment with luminal stenosis (marked with an arrow) by DSA criteria was shown. Corresponding segment on 2D CTA in short axis and in long axis were shown at the position with the greatest degree of the stenosis. Corresponding segment on 3D CTA datasets which were reconstructed from 2D CTA datasets by both short axis and long axis were also shown.

Lesion and reference segment characteristics and the correlation coefficients of anatomic measurements between CTA and DSA are listed in Table 2. As shown in the table, the results of Pearson correlation coefficients of anatomic measurements between CTA and DSA showed that 3D CTA-derived \%DS had the strongest significant correlation with DSA ( $\mathrm{r}=0.74, \mathrm{P}<0.001)$, followed by \%AS $(\mathrm{r}=0.67, \mathrm{P}<0.001)$, and then MLD $(\mathrm{r}=0.65, \mathrm{P}<0.001)$, and the MLA $(\mathrm{r}=0.51, \mathrm{P}<0.001)$.

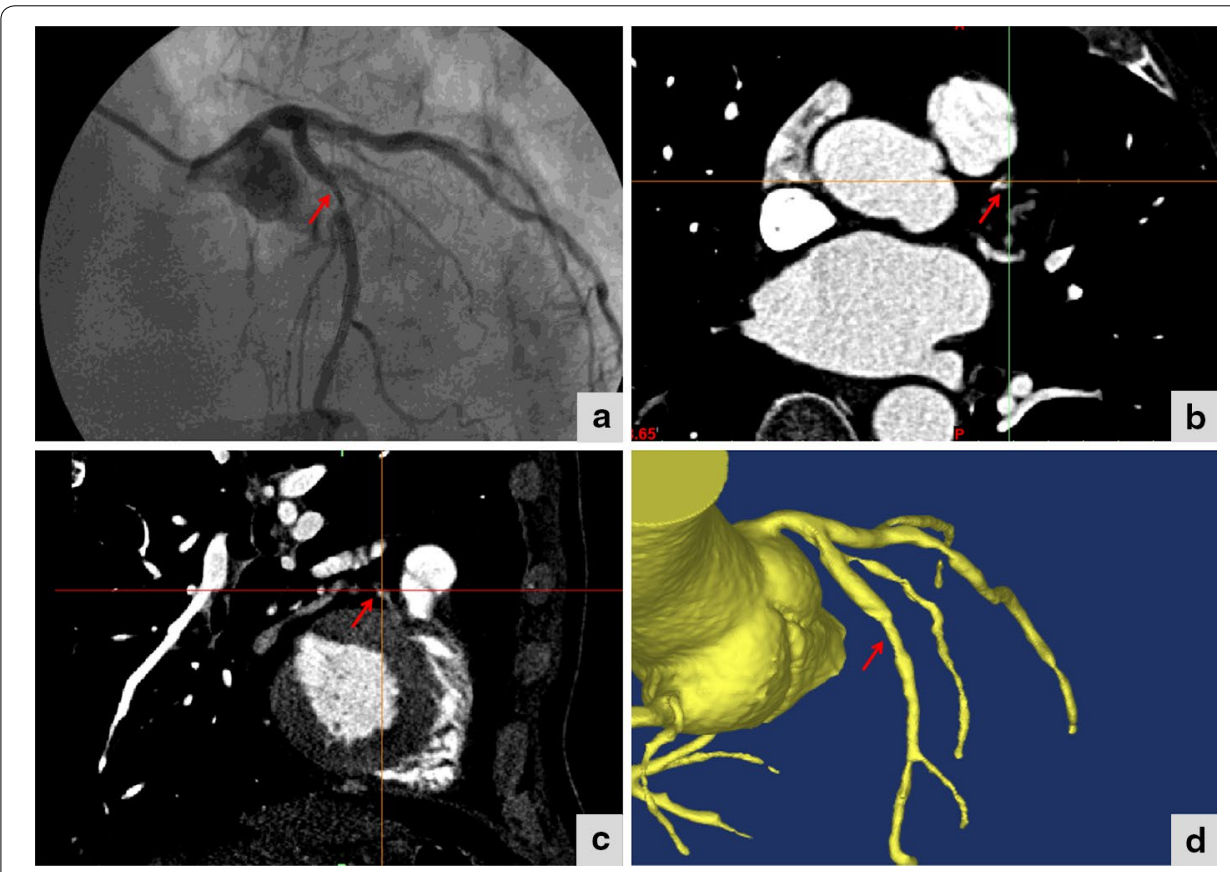

Figure 1 DSA, 2D CTA, and 3D CTA datasets of the same luminal stenosis. A coronary arterial segment with luminal stenosis (marked with an arrow) by DSA criteria is shown (a). Corresponding segment on 2D CTA in short axis (b) and in long axis (c) is shown at the position with the greatest degree of the stenosis. Corresponding segment on 3D CTA datasets (d).

Table 2 Lesion and reference segment characteristics

\begin{tabular}{lccll}
\hline Variables & DSA & 3D CTA & Pearson correlation & P \\
\hline MLD lesion $(\mathrm{mm})$ & $1.7 \pm 0.8$ & $1.6 \pm 0.7$ & 0.65 & $<0.001$ \\
Mean reference diameter $(\mathrm{mm})$ & $3.2 \pm 0.9$ & $2.7 \pm 0.7$ & 0.59 & $<0.001$ \\
\%DS & $47.4 \pm 20.9$ & $42.4 \pm 17.4$ & 0.74 & $<0.001$ \\
MLA lesion $\left(\mathrm{mm}^{2}\right)$ & $2.9 \pm 2.4$ & $3.1 \pm 2.2$ & 0.51 & $<0.001$ \\
Mean reference area & $8.5 \pm 5.1$ & $7.2 \pm 3.4$ & 0.620 & $<0.001$ \\
\%AS & $68.1 \pm 21.4$ & $59.4 \pm 19.7$ & 0.67 & $<0.001$ \\
\hline
\end{tabular}

$\mathrm{P} \leq 0.05$ was considered statistically significant. 
To illustrate the linear relationship more intuitionally, scatterplots with regression lines of MLD, \%DS, MLA, and \%AS between QCA and 3D CTA are shown Figure 2. The scatterplots confirmed the significant correlation between QCA and 3D CTA for the MLD, \%DS, MLA, and \%AS.

The results of Bland-Altman analysis are shown in Table 3 and in Figure 3 . The results illustrated small mean differences $(-1.11,-4.94,27.39$, and $-8.78 \%$ for MLD, \%DS, MLA, and \%AS, respectively) with relatively wide limits of agreement $( \pm 77.72, \pm 28.07$, $\pm 138.64, \pm 32.60 \%$ for MLD, \%DS, MLA, and \%AS, respectively).

The diagnostic performances of 3D CTA-derived anatomic parameters (\%DS, \%AS) for the detection of severe coronary arterial stenosis with DSA as the standard of reference are listed in Table 4.

The diagnostic accuracy was $74.1 \%$, the sensitivity was $58.3 \%$, the specificity was $86.7 \%$, the positive predictive value was $77.8 \%$, the negative predictive value was $91.7 \%$, and the receiver operating characteristic curve (ROC) was 0.73 for 3D CTA-derived \%DS for the detection of vessels with severe coronary arterial stenosis using DSA as the standard of reference. For 3D CTA-derived \%AS, the diagnostic accuracy was $74.1 \%$, the sensitivity

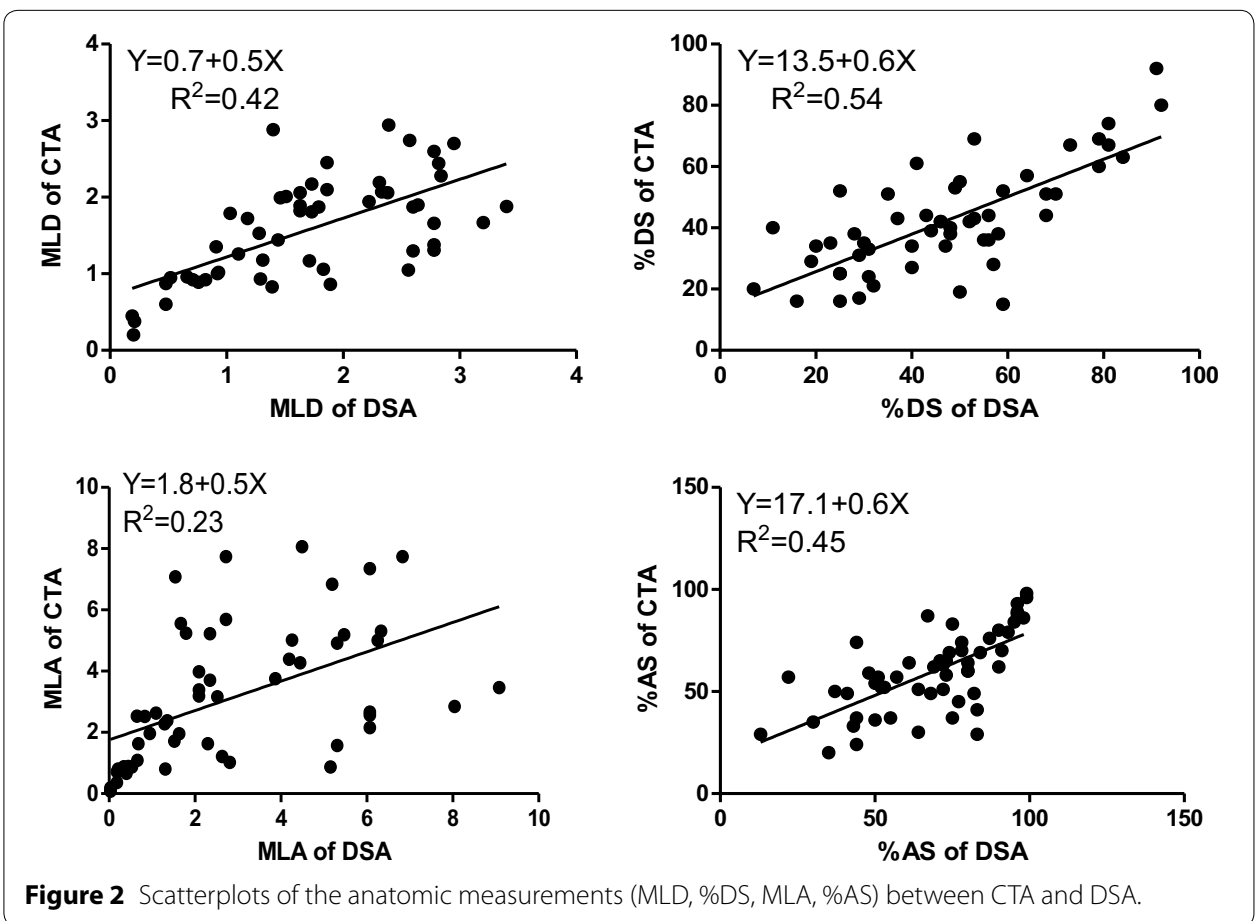

Table 3 Bland-Altman analysis of the anatomic measurements between 3D CTA and DSA

\begin{tabular}{lllll}
\hline Variables & $\begin{array}{l}\text { Mean difference } \\
\text { (absolute) }\end{array}$ & $\begin{array}{l}\text { Limits of agreement } \\
\text { (absolute) }\end{array}$ & $\begin{array}{l}\text { Mean difference } \\
(\%)\end{array}$ & $\begin{array}{l}\text { Limits of agreement } \\
\text { (\%) }\end{array}$ \\
\hline MLD & -0.13 & \pm 1.29 & -1.11 & \pm 77.72 \\
$\% D S$ & $-4.94 \%$ & $\pm 28.07 \%$ & -7.90 & \pm 79.05 \\
MLA & 0.26 & \pm 4.48 & 27.39 & \pm 138.64 \\
$\% A S$ & $-8.78 \%$ & $\pm 32.60 \%$ & -13.16 & \pm 65.03 \\
\hline
\end{tabular}




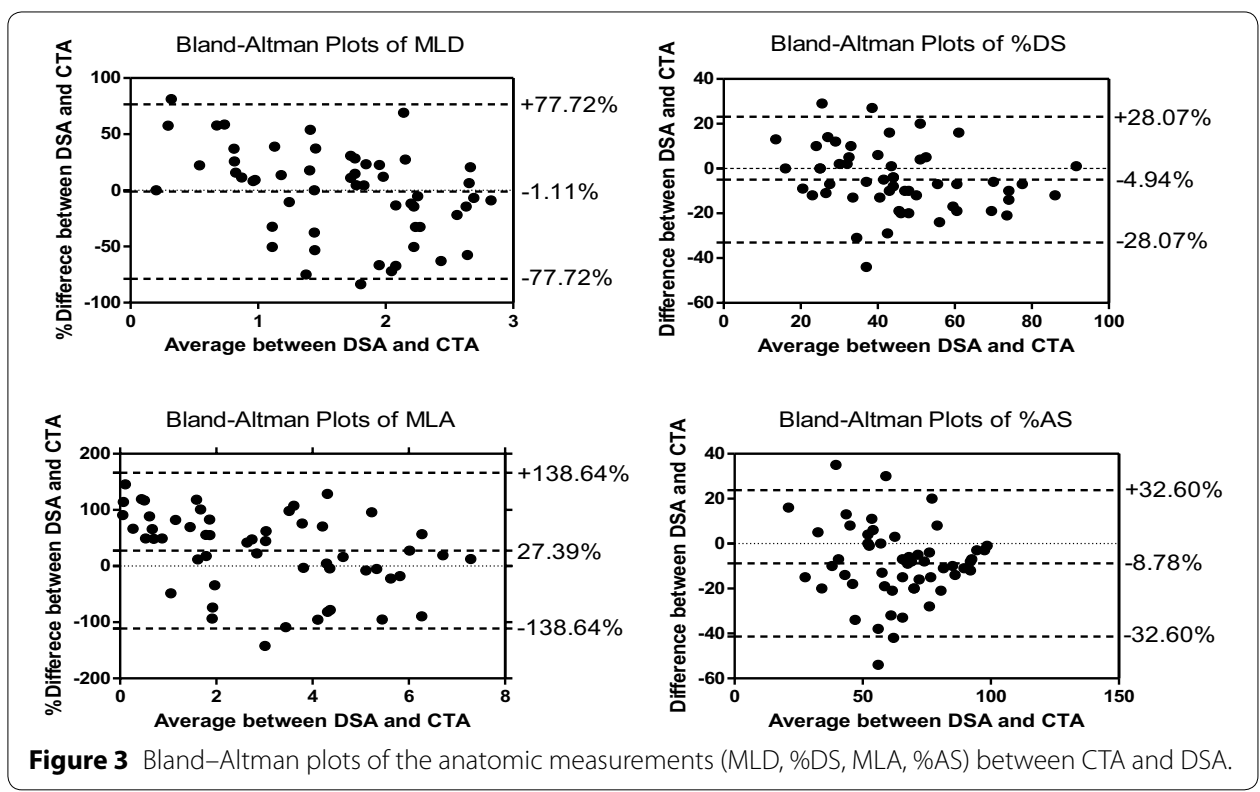

Table 4 The diagnostic performances of 3D CTA-derived anatomic parameters (\%DS, \%AS) for the detection of severe coronary arterial stenosis (as assessed by DSA)

\begin{tabular}{llllllllllll}
\hline & TP & TN & FP & FN & Kappa & $\begin{array}{l}\text { Sen } \\
\text { (\%) }\end{array}$ & $\begin{array}{l}\text { Spe } \\
\text { (\%) }\end{array}$ & $\begin{array}{l}\text { PPV } \\
\text { (\%) }\end{array}$ & $\begin{array}{l}\text { NPV } \\
\text { (\%) }\end{array}$ & $\begin{array}{l}\text { Diagnostic } \\
\text { accuracy (\%) }\end{array}$ & ROC area \\
\hline \%DS & 14 & 26 & 4 & 10 & 0.46 & 58.3 & 86.7 & 77.8 & 91.7 & 74.1 & 0.73 \\
\hline AS & 11 & 29 & 1 & 13 & 0.45 & 45.8 & 96.7 & 72.2 & 69.0 & 74.1 & 0.71 \\
\hline
\end{tabular}

was $45.8 \%$, the specificity was $96.7 \%$, the positive predictive value was $72.2 \%$, the negative predictive value was $69.0 \%$, and the ROC was 0.71 . The diagnostic concordance by 3D CTA-, and DSA-derived \%DS for the detection of vessels with and without severe stenosis was moderate (kappa value of 0.46 ); and for 3D CTA- and DSA-derived \%AS, the result was also moderate (kappa value of 0.45 ).

\section{Discussion}

This study was to investigate the accuracy of 3D quantitative CTA for the assessment of coronary stenosis using DSA as the standard of reference in patients with interpretable coronary arterial stenosis. The key finding of this study was that the statistical correlation between 3D CTA-derived anatomic parameters (MLD, \%DS, MLA, \%AS) and corresponding anatomic parameters derived from DSA were significant $(r=0.51-0.74, \mathrm{P}<0.001)$, and the Bland-Altman analysis confirmed that the mean differences was small (from -1.11 to $27.39 \%)$.

In our study, the correlations of the anatomic parameters between CTA and DSA were significant. The Pearson correlation coefficients were $0.74,0.67,0.65,0.51$ for \%DS, \%AS, MLD, and MLA, respectively. It is similar to a previous study, which also used 3D CTA technique for the quantification of coronary stenosis. They found significant correlation between CTA- and IVUS-derived anatomic parameters $(r=0.41-0.84, \mathrm{P}<0.001)$ [24]. 
These results are better than two previous studies, which use traditional one cross-sectional slice for stenosis analysis [21, 22]. In those studies, Meijboom et al. [21] evaluated 89 lesions in 79 patients by CTA and QCA. The subjects were scanned with a 64-slice CT scanner or a dual-source CT scanner. The correlation of the \%DS as determined by CTA and QCA was significant, but moderate $(\mathrm{R}=0.53$; $\mathrm{p}<0.001)$. Joshi et al. [22] evaluated 67 lesions in 55 patients by CTA and DSA. The CTA scans were performed on a 64-slice scanner. The results showed that the correlation between CTA- and QCAderived minimal luminal diameter (MLD) or diameter stenosis (DS) were not significant. That is, for MLD, $\mathrm{r}^{2}<0.01, \mathrm{P}=0.57$; and for DS, $\mathrm{r}^{2}=0.02, \mathrm{P}=0.31$. Therefore, 3D CTA technique shows great potential for improving the accuracy of evaluating coronary stenosis. That maybe because that 3D technique can be used for the analysis of the entire vessel segment, while traditional cross-sectional slice can only provide information in two views. Another reason for the improvement of the correlation was the used of the more advanced scanner.

The diagnostic accuracy was moderate in this study (74.1\% with $58.3 \%$ sensitivity and 86.7\% specificity for DS\%; $74.1 \%$ with $45.8 \%$ sensitivity and $96.7 \%$ specificity for $\% A S$ ), which was lower than a previous study [4]. In that study, CTA image sequences were also $3 \mathrm{D}$ reconstructed by software, whereas the positive predictive value of CTA in detection of coronary artery significant stenosis was $94 \%$ (with $100 \%$ sensitivity and $92 \%$ specificity). That maybe because of the more advanced scanner used in that study (320-slice CT scanner). In our study, CT image series were captured by 256-slice spiral CT scanner. In addition, in our experiment, we noticed that calcifications can obscure the lumen in CTA images, which lead to the overestimation of the stenosis severity when compared with DSA. It is as similar as its influence to 2D CTA [30-32]. Therefore, further study can investigate more details of the accuracy of stenosis assessment using 3D CTA in subjects with different degrees of calcification.

A limitation of this study is that the sample size is relativity small, and all the subjects are from a single clinical center. Validation with large sample size and multi center is needed before the clinical application of using 3D CTA as an alternative to assess luminal stenosis. Another limitation of this study is that the diagnostic performance in the detection of severe coronary stenosis was moderate. That may be improved by the use of more advanced CT technique. The CT scanner is 256-slice in our study, however, more advanced CT technique is available nowadays, such as 320 -slice.

\section{Conclusions}

In conclusion, our study validated the use of noninvasive 3D CTA for anatomic assessment of stenosis. We confirmed that anatomic assessments of stenosis evaluated by 3D CTA had significant statistical correlation with those evaluated by DSA, and the mean differences were small. It shows great potential for improving the accuracy of evaluating coronary stenosis. However, the diagnostic performance of using 3D CTA for the detection of severe coronary lesion is still with respected to be improved as the improvement of spatial and temporal resolution of CT scanner. It is respected that $3 \mathrm{D}$ advanced imaging reconstruction technique can be a helpful tool to promote the use of CTA as an alternative to assess luminal stenosis in patients presenting with chest pain syndromes. 


\section{Abbreviations}

2D: two-dimensional; 3D: three-dimensional; CTA: computed tomography angiography; DSA: digital subtraction angiography; MLD: minimal lumen diameter; MLA: minimal lumen area; \%DS: percent diameter stenosis; \%AS: percent area stenosis; CAD: coronary artery disease; PRefD: proximal reference diameter; DRefD: distal reference diameter; PRefA: proximal reference area; TP: true positive; TN: true negative; FP: false positive; FN: false negative; SBP: systolic blood pressure; DRefA: distal reference area; LDL: low-density lipoprotein cholesterol; TC: total cholesterol; CABG: coronary artery bypass graft; LAD: left anterior descending artery; RCA: right coronary artery; LCX: left circumflex artery; LM: left main coronary artery; D1: the first diagonal; OM1: the first obtuse marginal; ROC: receiver operating characteristic curve; Sen: sensitivity; Spe: specificity; PPV: positive predictive value; NPV: negative predictive value; MI: myocardial infarction; QCA: quantitative coronary angiography.

\section{Authors' contributions}

YT Zhang initiated and supported this project. W Guo, X Liu, ZF Gao, and WH Lin participated in the design of the study. $X$ Lin and WH Lin participated in the work of simulation and provided technical supports. WH Lin and HY Zhang drafted the manuscript. W Guo, WH Lin, and N Tan carried out the clinical examination and provided clinical supports, S Pirbhulal checked through all the writing. All authors read and approved the final manuscript.

\section{Author details}

1 Department of Cardiology, Guangdong Cardiovascular Institute, Guangdong General Hospital, Guangdong Academy of Medical Sciences, Guangzhou, China. ${ }^{2}$ Institute of Biomedical and Health Engineering, Shenzhen Institutes of Advanced Technology, Shenzhen 518055, China. ${ }^{3}$ Key Lab for Health Informatics, Chinese Academy of Sciences, Shenzhen 518055, China. ${ }^{4}$ Institute of Clinical Anatomy, Southern Medical University, Guangzhou, China. ${ }^{5}$ Department of Electronic Engineering, The Joint Research Centre for Biomedical Engineering, Chinese University of Hong Kong, Hong Kong, China. ${ }^{6}$ Shenzhen College of Advanced Technology, University of Chinese Academy of Sciences, Beijing, China.

\section{Acknowledgements}

This work was supported in part by Guang-Dong Innovation Research Team Fund for Low-cost Health-care Technologies in China, the Key Lab for Health Informatics of the Chinese Academy of Sciences, the Enhancing Program of Key Laboratories of Shenzhen City (ZDSY20120617113021359), the National High-tech R\&D Program (863 Program) (No. 2012AA02A603), the Shenzhen Innovation Funding (ZDSY20120617113021359, JCYJ20140414170821190, SGLH20131010110119871, GJHZ20140415152115754).

\section{Compliance with ethical guidelines}

\section{Competing interests}

The authors declare that they have no conflict of interest.

Received: 8 January 2015 Accepted: 7 May 2015

Published online: 30 May 2015

\section{References}

1. Dewey M, Rutsch W, Schnapauff D, Teige F, Hamm B. Coronary artery stenosis quantification using multislice computed tomography. Invest Radiol. 2007;42(2):78-84.

2. Nakazato R, Shalev A, Doh JH, Koo BK, Gransar H, Gomez MJ, et al. Aggregate plaque volume by coronary computed tomography angiography is superior and incremental to luminal narrowing for diagnosis of ischemic lesions of intermediate stenosis severity. J Am Coll Cardiol. 2013;62(5):460-7.

3. Bucek RA, Puchner S, Haumer M, Reiter M, Minar E, Lammer J. CTA quantification of internal carotid artery stenosis: application of luminal area vs. luminal diameter measurements and assessment of inter-observer variability. J Neuroimaging. 2007;17(3):219-26.

4. Youssef MA, Dawoud MA, Elbarbary AA, Elbedewy MM, Elkhateeb HM. Role of 320-slice multislice computed tomography coronary angiography in the assessment of coronary artery stenosis. Egypt J Radiol Nucl Med. 2014;45(2):317-24

5. Neefjes LA, de Feyter PJ. CT coronary angiography: a new unique prognosticator? Heart. 2011;97(17):1363-4

6. Kopp AF, Kuttner A, Trabold T, Heuschmid M, Schroder S, Claussen CD. Multislice CT in cardiac and coronary angiography. Br J Radiol. 2004;77(1):87-97.

7. Gitsioudis G, Katus HA, Korosoglou G. Assessment of coronary artery disease using coronary computed tomography angiography and biochemical markers. World J Cardiol. 2014;6(7):663-70.

8. Sun Z, Lin C, Davidson R, Dong C, Liao Y. Diagnostic value of 64-slice CT angiography in coronary artery disease: a systematic review. Eur J Radiol. 2008;67(1):78-84.

9. Sun Z, Almutairi AM. Diagnostic accuracy of 64 multislice CT angiography in the assessment of coronary in-stent restenosis: a meta-analysis. Eur J Radiol. 2010;73(2):266-73.

10. Munnur RK, Cameron JD, Ko BS, Meredith IT, Wong DT. Cardiac CT: atherosclerosis to acute coronary syndrome Cardiovasc Diagn Ther. 2014;4(6):430-48.

11. Fischer C, Hulten E, Belur P, Smith R, Voros S, Villines TC. Coronary CT angiography versus intravascular ultrasound for estimation of coronary stenosis and atherosclerotic plaque burden: a meta-analysis. J Cardiovasc Comput Tomogr. 2013;7(4):256-66.

12. Naganuma T, Latib A, Costopoulos C, Takagi K, Naim C, Sato K, et al. The role of intravascular ultrasound and quantitative angiography in the functional assessment of intermediate coronary lesions: correlation with fractional flow reserve. Cardiovasc Revascularization Med. 2014;15(1):3-7.

13. Chang SM, Bhatti S, Nabi F. Coronary computed tomography angiography. Curr Option Cardiol. 2011;26(5):392-402. 
14. Voros S, Joshi P, Qian Z, Rinehart S, Vazquez-figueroa JG, Anderson H, et al. Apoprotein B, small-dense LDL and impaired HDL remodeling is associated with larger plaque burden and more noncalcified plaque as assessed by coronary CT angiography and intravascular ultrasound with radiofrequency backscatter: results from the Atlanta I study. J Am Heart Assoc. 2013;2(6):e000344.

15. Sun Z. Evidence for myocardial CT perfusion imaging in the diagnosis of hemodynamically significant coronary artery disease. Cardiovas Diagn Ther. 2015;5(1):58-62.

16. Zarins CK, Taylor CA, Min JK. Computed fractional flow reserve (FFTCT) derived from coronary CT angiography. J Cardiovasc Transl Res. 2013;6(5):708-14.

17. Kang SJ, Ahn JM, Han S, Lee JY, Kim WJ, Park DW, et al. Sex differences in the visual-functional mismatch between coronary angiography or intravascular ultrasound versus fractional flow reserve. Jacc Cardiovasc Interv. 2013;6(6):562-8.

18. Xu L, Sun Z. Coronary CT angiography evaluation of calcified coronary plaques by measurement of left coronary bifurcation angle. Int J Cardiol. 2015;182:229-31.

19. de Graaf MA, Broersen A, Ahmed W, Kitslaar PH, Dijkstra J, Kroft LJ, et al. Feasibility of an automated quantitative computed tomography angiography-derived risk score for risk stratification of patients with suspected coronary artery disease. Am J Cardiol. 2014;113(12):1947-55.

20. de Graaf MA, Broersen A, Kitslaar PH, Roos CJ, Dijkstra J, Lelieveldt BP, et al. Automatic quantification and characterization of coronary atherosclerosis with computed tomography coronary angiography: cross-correlation with intravascular ultrasound virtual histology. Int J Cardiovasc Imaging. 2013;29(5):1177-90.

21. Meijboom WB, van Mieghem CA, van Pelt N, Weustink A, Pugliese F, Mollet NR, et al. Comprehensive assessment of coronary artery stenoses: computed tomography coronary angiography versus conventional coronary angiography and correlation with fractional flow reserve in patients with stable angina. J Am Coll Cardiol. 2008;52(8):636-43.

22. Joshi SB, Okabe T, Roswell RO, Weissman G, Lopez CF, Lindsay J, et al. Accuracy of computed tomographic angiography for stenosis quantification using quantitative coronary angiography or intravascular ultrasound as the gold standard. Am J Cardiol. 2009;104(8):1047-51.

23. Sarno G, Decraemer I, Vanhoenacker PK, de Bruyne B, Hamilos M, Cuisset T, et al. On the inappropriateness of noninvasive multidetector computed tomography coronary angiography to trigger coronary revascularization: a comparison with invasive angiography. Jacc Cardiovasc Interv. 2009;2(6):550-7.

24. Voros S, Rinehart S, Vazquez-Figueroa JG, Kalynych A, Karmpaliotis D, Qian Z, et al. Prospective, head-to-head comparison of quantitative coronary angiography, quantitative computed tomography angiography, and intravascular ultrasound for the prediction of hemodynamic significance in intermediate and severe lesions, using fractional flow reserve as reference standard (from the Atlanta I and II study). Am J Cardiol. 2014;113(1):23-9.

25. Voros S, Rinehart S, Qian Z, Vazquez G, Anderson H, Murrieta L, et al. Prospective validation of standardized, 3-dimensional, quantitative coronary computed tomographic plaque measurements using radiofrequency backscatter intravascular ultrasound as reference standard in intermediate coronary arterial lesions: results from the Atlanta (assessment of tissue characteristics, lesion morphology, and hemodynamics by angiography with fractional flow reserve, intravascular ultrasound and virtual histology, and noninvasive computed tomography in atherosclerotic plaques) I study. Jacc Cardiovasc Interv. 2011;4(2):198-208.

26. Rinehart S, Vazquez G, Qian Z, Voros S. Coronary plaque imaging with multi-slice computed tomographic angiography and intravascular ultrasound: a close look inside and out. J Invasive Cardiol. 2009;21(7):367-72.

27. Rinehart S, Vazquez G, Qian Z, Murrieta L, Christian K, Voros S. Quantitative measurements of coronary arterial stenosis, plaque geometry, and composition are highly reproducible with a standardized coronary arterial computed tomographic approach in high-quality CT datasets. J Cardiovasc Comput Tomogr. 2011;5(1):35-43.

28. Li JL, Huang MP, Liang CH, Zhao ZJ, Liu H, Cui YH, et al. Individualized radiation dose control in 256-slice CT coronary angiography (CTCA) in retrospective ECG-triggered helical scans: using a measure of body size to adjust tube current selection. Eur J Radiol. 2012;81(11):3146-53.

29. Bland JM, Altman DG. Statistical methods for assessing agreement between two methods of clinical measurement. Lancet. 1986;1 (8476):307-10.

30. Arbab-Zadeh A, Hoe J. Quantification of coronary arterial stenoses by multidetector $\mathrm{CT}$ angiography in comparison with conventional angiography methods, caveats and implications. Jacc Cardiovasc Imaging. 2011;4(2):191-202.

31. Vavere AL, Arbab-Zadeh A, Rochitte CE, Dewey M, Niinuma H, Gottlieb I, et al. Coronary artery stenoses: accuracy of 64-detector row ct angiography in segments with mild, moderate, or severe calcification-a subanalysis of the core-64 trial. Radiology. 2011;261(1):100-8.

32. Schoepf UJ, Zwerner PL, Savino G, Herzog C, Kerl JM, Costello P. Coronary CT angiography. Radiology. 2007;244(1):48-63.

\section{Submit your next manuscript to BioMed Central and take full advantage of:}

- Convenient online submission

- Thorough peer review

- No space constraints or color figure charges

- Immediate publication on acceptance

- Inclusion in PubMed, CAS, Scopus and Google Scholar

- Research which is freely available for redistribution

Submit your manuscript at

www.biomedcentral.com/submit

( ) Biomed Central 